CAPÍTULO III

\title{
TECNOLOGÍAS DE LA INFORMACIÓN Y LAS COMUNICACIONES - TIC
}

Autor: Andrés Ignacio Zamudio Castro

\section{OBJETIV0}

"Las Tecnologías de la Información y de las Comunicaciones (TIC) son un elemento articulador entre los procesos de producción, distribución y disposición final en la cadena de abastecimiento, vinculando adicionalmente a los diferentes actores involucrados en los flujos de bienes e información tanto en la solicitud y recepción de pedidos, órdenes de servicios, transporte y almacenamiento de los bienes"

- (CONPES 3547). 
Teniendo en cuenta la definición anterior, en la cual se establece y se evidencia la importancia de las Tecnologías de la Información y de las Comunicaciones en la articulación de las actividades logísticas a nivel nacional, resulta comprensiva su categorización como uno de los subsistemas que se deben medir y evaluar para comparar el desempeño de la política nacional logística.

Adicionalmente, se ha evidenciado que la implementación de las Tecnologías de la Información y de las Comunicaciones en las organizaciones genera cambios en las estructuras y en las operaciones organizacionales, mejorando la eficiencia, reduciendo los costos e incrementando los ingresos.

Por otra parte, atendiendo la participación de Colombia en múltiples tratados comerciales, se evidencia el alto nivel de competitividad asociado con el comercio exterior. Esto requiere que se adopten nuevos mecanismos que permitan asegurar la información de las operaciones logísticas y, al mismo tiempo, permitan tener un claro conocimiento sobre los flujos transversales en las cadenas de suministro y abastecimiento.

\section{ALCANCE}

El alcance asociado con el subsistema de las Tecnologías de la Información y Comunicación se desarrolla en la generación de indicadores que permitan medir el desempeño de las variables y las políticas impulsadas por el gobierno nacional en materia de TIC. El objetivo de dichos indicadores está relacionado con el desarrollo e implementación de sistemas de información que permitan realizar el monitoreo del transporte de carga, impulsando actividades tales 
como tareas analíticas vinculadas a la caracterización de los ciclos y operaciones logísticas de transporte de carga, las relaciones entre los diferentes agentes logísticos que intervienen en la cadena. Dichos indicadores se deberán alimentar de nuevos sistemas de información o de los sistemas existentes con los que actualmente cuenta el sector logístico, como por ejemplo:

- Registro Nacional de Despachos de Carga (RNDG)

- Modelo de Oferta Vehicular (MOV)

- Índice de Costos del Transporte de Carga por Carretera (ICTC)

- Sistema de Información de Costos Eficientes (SICE TAC)

- Sistema de Información para la Regulación del Transporte de Carga por Carretera (SIRTCG)

\section{ANTECEDENTES}

De acuerdo con el documento 3547 generado por el Consejo Nacional de Política Económica y Social (CONPES), del Departamento Nacional de Planeación de la república de Colombia, "las políticas para el sector productivo estarán orientadas a incrementar el uso y apropiación de las Tecnologías de la Información y de las Comunicaciones en las empresas con el fin último de contribuir al aumento de la competitividad, y por ende a la productividad empresarial". 
Desde este punto de vista, el Gobierno Nacional busca promover la integración operacional entre los diferentes subsistemas (Urbano Territorial, Transporte e Infraestructura y Servicios Logísticos), medidos a partir de las diferentes categorías utilizadas para la evaluación de los sistemas logísticos a nivel nacional (Tiempos, Costos, Confiabilidad y Flexibilidad $)^{2}$ con las TIC, donde estas serán el eje articulador de las actividades logísticas a nivel nacional. Desde este punto de vista, lo que se busca es que todos los actores de las cadenas logísticas realicen sus operaciones bajo un ambiente confiable, predecible y eficiente, donde los flujos de información en las cadenas logísticas sean visibles y transparentes para cada uno de los actores involucrados, generando conectividad virtual y visibilidad de los procesos, permitiendo operaciones continuas, eficientes, seguras y de calidad, garantizando que los costos generados en dichas operaciones sean mínimos.

La importancia que le ha otorgado el Gobierno Nacional a las Tecnologías de la Información y de las Comunicaciones no solo se ha visto resaltada en el CONPES. Como uno de los ejes centrales del Plan Nacional de Tecnologías de la Información y de las Comunicaciones (PNTIC) 2008-2019, se busca promover la utilización de los sistemas de información y de comunicaciones en el sector productivo con la participación de las entidades públicas y privadas en los diferentes sectores empresariales.

Particularmente, la vinculación de las Tecnologías de la Información y de las Comunicaciones como eje articulador de los procesos logísticos está orientada al incremento de la productividad nacional, por medio de sistemas eficientes que permitan el

2 Tomado de "Best Practices In Corridor Managemet". Trade LogisTIC Group - The World Bank. 
- intercambio comercial de bienes y mercancías y el monitoreo de dichas actividades. Por lo tanto, se deberán diseñar, evaluar e implementar tecnologías cuyo objetivo sea:

- Sistemas de datawarehouse (almacenamiento y recuperación de datos)

- Rastreo y posicionamiento a mercancías y vehículos de transporte.

- Sistemas web para la consolidación de la oferta y demanda de servicios logísticos.

- Sistemas web para la consolidación de transacciones comerciales.

- "Sistemas enfocados a la simplificación de procedimientos administrativos ante autoridades de control de las operaciones de comercio exterior"3.

\section{PLAN NACIONAL DE TECNOLOGÍAS DE LA INFORMACIÓN Y LAS COMUNICACIONES (PNTIC) 2008-2019}

El Plan Nacional de Tecnologías de la Información y las Comunicaciones (PNTIC) es una "política de estado que tiene como principal objetivo contribuir a la inclusión social y la competitividad

3 Tomado de CONPES 3547. Departamento Nacional de Planeación (DNP). Octubre de 2008. 
del país, a través de estrategias que mejoren el uso y la apropiación de las tecnologías de la información y las comunicaciones (TIC)"4.

Dicho plan se concentra en ocho ejes o pilares básicos. De estos ocho ejes, cuatro se consideran ejes transversales, ya que cubren aspectos y programas que generan un impacto directo sobre la sociedad y la economía. Dichos ejes son:

- Comunidad - Acceso masificado a las TIC.

- Gobierno en línea.

- Investigación, desarrollo e innovación.

- Marco regulatorio e incentivos.

Los cuatro ejes restantes se consideran verticales, ya que se centran en sectores estratégicos, caracterizados así por el PNTIC. Dichos ejes son:

- Educación - incorporación de las TIC.

- Salud.

- Justicia.

- Competitividad Empresarial - Acciones orientadas a generar desarrollos en los sectores productivos del país.

4 Tomado de CONPES 3779. Departamento Nacional de Planeación (DNP). Octubre de 2013. 


\section{ACTORES DE LAS TECNOLOGÍAS DE LA INFORMACIÓN Y LAS}

COMUNICACIONES - TIC

Los principales actores del subsistema de las Tecnologías de la Información y de las Comunicaciones (TIC) son un conjunto de entidades gubernamentales y privadas, las cuales serán las encargadas de generar y suministrar la información de forma oportuna que alimentará el conjunto de indicadores desarrollados para la evaluación y el monitoreo del subsistema descrito.

Los actores relevantes para dicho objetivo son: 


\section{Actores gubernamentales}

\begin{tabular}{c|c} 
ACTOR & ¿CÓMO INCIDE EL ACTOR EN EL SISTEMA? \\
\hline Ministerio de & $\begin{array}{c}\text { Formulación y adopción de las políticas, planes, programas, } \\
\text { proyectos y regulación económica en materia de transporte, } \\
\text { tránsito e infraestructura de los modos de transporte carre- } \\
\text { tero, marítimo, fluvial, férreo y aéreo y la regulación técnica } \\
\text { en materia de transporte y tránsito de los modos carretero, } \\
\text { marítimo, fluvial y férreo. }\end{array}$ \\
\hline \multirow{2}{*}{$\begin{array}{c}\text { El Instituto Nacional de Vías - INVIAS es una agencia a cargo } \\
\text { de la asignación, regulación y supervisión de los contratos para } \\
\text { la construcción de carreteras y su respectivo mantenimiento. }\end{array}$}
\end{tabular}

Entre las principales responsabilidades, se puede mencionar:

1. Ejecuta la política del Gobierno Nacional en relación con la infraestructura de su competencia, de conformi-

Instituto Na-

cional de Vías

- INVIAS dad con los lineamientos establecidos por el Ministerio de Transporte.

2. Elabora conjuntamente con el Ministerio de Transporte los planes, programas y proyectos tendientes a la construcción, reconstrucción, mejoramiento, rehabilitación, conservación, atención de emergencias, y demás obras que requiera la infraestructura de su competencia.

3. Coordina con el Ministerio de Transporte la ejecución de los planes y programas de su competencia.

4. Adelanta investigaciones, estudios, y supervisa la ejecución de las obras de su competencia conforme a los planes y prioridades nacionales. 


\begin{tabular}{|c|c|}
\hline ACTOR & ¿CÓMO INCIDE EL ACTOR EN EL SISTEMA? \\
\hline & $\begin{array}{l}\text { "La Agencia Nacional de Infraestructura - ANI, es una agencia } \\
\text { gubernamental y tiene por objeto planear, coordinar, estruc- } \\
\text { turar, contratar, ejecutar, administrar y evaluar proyectos de } \\
\text { concesiones y otras formas de Asociación Público Privada - } \\
\text { APP, para el diseño, construcción, mantenimiento, operación, } \\
\text { administración y/o explotación de la infraestructura pública de } \\
\text { transporte en todos sus modos y de los servicios relacionados" }\end{array}$ \\
\hline
\end{tabular}

Agencia Na-

cional de In-

fraestructura

- ANI

Entre sus responsabilidades más relevantes se encuentran:

1. Identificar, evaluar la viabilidad y proponer iniciativas de concesión u otras formas de Asociación Público Privada para el desarrollo de la infraestructura de transporte y de los servicios relacionados.

2. Elaborar los estudios para definir los peajes, tasas, tarifas, contribución de valorización y otras modalidades de retribución por el diseño, construcción, operación, explotación, mantenimiento o rehabilitación de la infraestructura relacionada con los proyectos de concesión u otras formas de Asociación Público Privada.

Ministerio de

Tecnologías

de la Infor-

mación y Co-

municaciones

- MINTIC
El Ministerio de Tecnologías de la Información y las Comunicaciones es la entidad que se encarga de diseñar, adoptar y promover las políticas, planes, programas y proyectos del sector de las Tecnologías de la Información y las Comunicaciones.

Dentro de sus funciones está:

1. Diseñar, formular, adoptar y promover las políticas, planes, programas y proyectos del Sector de Tecnologías de la de la Información y, en correspondencia con la Constitución Política y la ley, con el fin de contribuir al desarrollo económico, social y político de la Nación y elevar el bienestar de los colombianos.

2. Impulsar el desarrollo y fortalecimiento del Sector de Tecnologías de la Información y las Comunicaciones, promover la investigación e innovación, buscando su competitividad y avance tecnológico conforme al entorno nacional e internacional. 


\section{Actores Privados}

\begin{tabular}{|c|c|}
\hline ACTOR & ¿CÓMO INCIDE EL ACTOR EN EL SISTEMA? \\
\hline $\begin{array}{l}\text { Operadores } \\
\text { de Transporte }\end{array}$ & $\begin{array}{l}\text { 1. Encargados del transporte de la carga desde los } \\
\text { puntos de origen a los puntos de destino de la } \\
\text { carga. } \\
\text { 2. Distribución y procesos de cargue y descargue. } \\
\text { 3. Velar por el adecuado transporte y su arribo a } \\
\text { tiempo }\end{array}$ \\
\hline $\begin{array}{l}\text { Operadores } \\
\text { Logísticos }\end{array}$ & $\begin{array}{l}\text { 4. Gestión y administración de plataformas logísticas } \\
\text { 5. Operación de servicios y terminales de carga. } \\
\text { 6. Promover las TIC como herramienta de control de } \\
\text { los flujos de información. }\end{array}$ \\
\hline
\end{tabular}




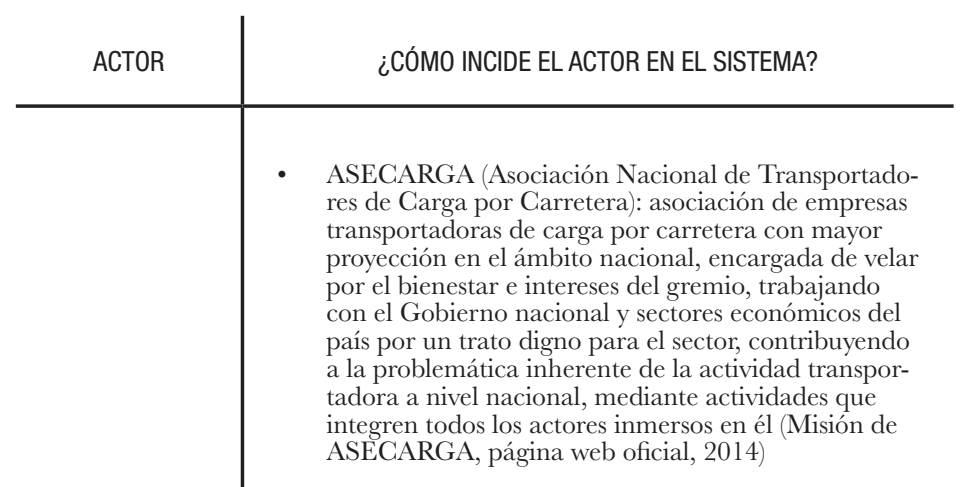

- COLFECAR: Federación Colombiana de Transportadores de Carga por Carretera: Desarrollar acciones gremiales con sentido de propuesta ante el Gobierno nacional.Intervenir propositivamente en la formulación del marco legal, estatutario y reglamentario de la actividad transportadora.

Velar por la promoción y desarrollo de los recursos humanos vinculados al sector transportador. Propiciar acuerdos con los usuarios para todos los fines de interés común y convivencia recíproca y coordinar a los federados en lo relativo a la explotación razo-

Agremiaciones

de Transporta-

dores - Sector

Privado nable de la actividad transportadora. Contribuir al desarrollo de programas de protección social para los afiliados (Objetivos de COLFECAR, página web oficial, 2014).

- ATC: Asociación de Transportadores de Carga: ejercer representación, vocería y asesoría al transporte de carga en territorio nacional e internacional, mediante estructura de una organización competente que disponga de recursos físicos y humanos para realizar investigación y propuestas comerciales en procura de la sostenibilidad y proyección de los transportadores de carga terrestres frente a los modelos económicos planteados en el país (Misión de la ATC, página web oficial, 2014).

- CGT: Confederación Colombiana de Transportadores.

- FEDETRANSCOL: Federación de Empresas Transportadoras de Carga por Carretera.

- ANDI: Asociación Nacional de Empresarios de Colombia.

- FENALCO: Federación Nacional de Comerciantes. 


\begin{tabular}{|c|c|}
\hline ACTOR & ¿CÓMO INCIDE EL ACTOR EN EL SISTEMA? \\
\hline & $\begin{array}{l}\text { El Ministerio de Tecnologías de la Información y las Comu- } \\
\text { nicaciones es la entidad que se encarga de diseñar, adoptar } \\
\text { y promover las políticas, planes, programas y proyectos del } \\
\text { sector de las Tecnologías de la Información y las Comu- } \\
\text { nicaciones. }\end{array}$ \\
\hline $\begin{array}{l}\text { Ministerio de } \\
\text { Tecnologías de }\end{array}$ & Dentro de sus funciones está: \\
\hline \multirow[t]{2}{*}{$\begin{array}{l}\text { la Información } \\
\text { y Comunica- } \\
\text { ciones - MIN- } \\
\text { TIC }\end{array}$} & $\begin{array}{l}\text { Diseñar, formular, adoptar y promover las políticas, } \\
\text { planes, programas y proyectos del Sector de Tecno- } \\
\text { logías de la de la Información y, en correspondencia } \\
\text { con la Constitución Política y la ley, con el fin de } \\
\text { contribuir al desarrollo económico, social y político } \\
\text { de la Nación y elevar el bienestar de los colombia- } \\
\text { nos. }\end{array}$ \\
\hline & $\begin{array}{l}\text { 2. Impulsar el desarrollo y fortalecimiento del Sector } \\
\text { de Tecnologías de la Información y las Comuni- } \\
\text { caciones, promover la investigación e innovación, } \\
\text { buscando su competitividad y avance tecnológico } \\
\text { conforme al entorno nacional e internacional. }\end{array}$ \\
\hline
\end{tabular}

\section{INDICADORES DEL SUBSISTEMA DE}

\section{TECNOLOGÍAS DE LA INFORMACIÓN Y}

\section{LAS COMUNICACIONES - TIC}

Los indicadores elaborados fueron desarrollados bajo la guía de formulación de indicadores del Departamento Nacional de Planeación y alineados con los objetivos estratégicos planteados inicialmente por el equipo de trabajo del proyecto E-Transcol, sobre el deber ser de la eficiencia de las Tecnologías de la Información y de las Comunicaciones (TIC) en las operaciones y actividades logísticas. 
Durante la etapa de diseño, se generaron un conjunto de indicadores los cuales fueron evaluados y depurados. Al mismo tiempo, y teniendo en cuenta diferentes variables, como la periodicidad de medición y la forma de calculo del indicador, se agrupó en un conjunto de familias denominadas Macro - Indicadores. Esto se realizó con el objetivo de obtener un conjunto de indicadores generales que permitieran evaluar el desempeño del subsistema de TIC, y que pudiera ser analizado de forma rápida y eficiente en un cuadro integral del mando, definiendo claramente los responsables del manejo de dicho ítem, así como la periodicidad de medición.

El conjunto de Macro Indicadores se presenta a continuación, con su respectiva descripción:

\section{Inversión media en TIC por tamaño de empresa del} sector logístico: este indicador se compone de dos elementos: las inversiones realizadas en TIC por clasificación de empresa y tamaño de la empresa (pyme, mediana o grande). Este indicador es de tipo cuantitativo y busca evaluar los recursos destinados a la implementación de TIC según el tamaño de la empresa y su cálculo esta basado en la ponderación de factores.

\section{Porcentaje de uso según tipología de TIC (GPS,} sistema de planificación, servicio de trazabilidad, sistemas transaccionales: Este indicador permite cuantificar en una escala relativa el uso de los diferentes tipos de TIC aplicados a las operaciones logísticas y busca evaluar el número de empresas que incluyen algún tipo de tecnología en sus operaciones. 
3. Vehículos con implementación de TIC para trazabilidad en entregas por corredor: este indicador permite cuantificar en una escala relativa el uso de los diferentes tipos de TIC aplicados a las operaciones logísticas y busca evaluar el número de vehículos que incluyen algún tipo de tecnología para la trazabilidad de las entregas.

4. Inversión anual en TIC (sector público + privado): este indicador permite cuantificar en una escala relativa la inversión anual realizada en TIC en los sectores públicos y privados frente a la inversión anual realizada por los sectores en operaciones logísticas.

5. Porcentaje de cobertura a nivel nacional para el monitoreo de mercancías: este indicador permite cuantificar en una escala relativa el nivel de cobertura a nivel nacional para el monitoreo de mercancías, dividido en los diferentes departamentos constituidos oficialmente.

6. Número de sistemas de información de servicios logísticos en operación: este indicador permite cuantificar en una escala absoluta el número de sistemas de información logísticos que se encuentran operando a nivel nacional, con el objetivo de evaluar la robustez de los sistemas logísticos a nivel nacional.

\section{Número de sistemas de información de servicios} logísticos desarrollados y en fase de prueba: este indicador permite cuantificar en una escala absoluta el número de sistemas de información logísticos que se encuentran en 
desarrollo y en fases de prueba, ya sea como complemento de los sistemas existentes o como nuevos sistemas de información logísticos.

8. Porcentaje de transacciones de consolidación de carga realizadas vía web: Este indicador permite cuantificar en una escala relativa la cantidad de procesos de consolidación de carga en los diferentes puntos de interconexión a nivel nacional.

\section{9. Índice de costos de transporte por medio de trans-} porte: este Indicador permite establecer un valor promedio de carga movilizada por cada uno de los modos de transporte existentes y utilizados en el territorio nacional.

A continuación, se presenta cada uno de estos Macro Indicadores, con su respectiva familia de indicadores utilizados para su resultado, así como las formas de cálculo, sus unidades de medida, la periodicidad y las entidades responsables de cada uno de estos:

\section{Inversión media en TIC por tamaño de empresa del} sector logístico: A continuación, se presenta la Información asociada a este indicador:

\begin{tabular}{c|l} 
Forma de cálculo del Macro Indicador & $\begin{array}{l}\text { Ponderación cualitativa de los el- } \\
\text { ementos que lo componen }\end{array}$ \\
\hline Unidad de medición del Macro Indicador & Porcentaje ponderado \\
\hline
\end{tabular}




\begin{tabular}{|c|c|}
\hline Forma de cálculo & $\begin{array}{l}\text { 1. Se debe promediar la } \\
\text { inversión total realizada } \\
\text { en TIC entre el número } \\
\text { de empresas del sector. } \\
\text { 2. Una vez obtenidos los } \\
\text { promedios, se realiza una } \\
\text { suma producto de los valo- } \\
\text { res promedio en inversión } \\
\text { entre el valor asignado a } \\
\text { cada grupo de empresas } \\
\text { por tamaño }\end{array}$ \\
\hline Unidad de medición & SCOP \\
\hline Viabilidad & SI \\
\hline Entidad que proporciona la información & INVIAS \\
\hline Periodicidad & Anual \\
\hline
\end{tabular}

Tabla 1 - Macro indicador 1 - Fuente: Propia

Entre las observaciones realizadas para la implementación de este Macro Indicador está determinar la manera como se le va a dar un valor cualitativo a los elementos que lo componen. Por lo tanto, la consolidación de este Macro Indicador, una vez obtenida la información, debe ser realizada por el Ministerio de Transporte.

\section{Porcentaje de uso según tipología de TIG (GPS,} sistema de planificación, servicio de trazabilidad, sistemas transaccionales: A continuación, se presenta la Información asociada a este indicador:

\begin{tabular}{l|l} 
Forma de cálculo del Macro Indicador & $\begin{array}{l}\text { Ponderación cualitativa de los el- } \\
\text { ementos que lo componen }\end{array}$ \\
\hline Unidad de medición del Macro Indicador & Porcentaje ponderado \\
\hline
\end{tabular}




\begin{tabular}{c|l}
\hline \multirow{2}{*}{ Forma de cálculo } & $\begin{array}{l}\text { Número de empresas con } \\
\text { utilización de sistemas GPS/ } \\
\text { Número de empresas totales }\end{array}$ \\
\cline { 2 - 2 } & $\begin{array}{l}\text { Número de empresas con } \\
\text { utilización de sistemas de plani- } \\
\text { ficación/Número de empresas } \\
\text { totales }\end{array}$ \\
\cline { 2 - 2 } & $\begin{array}{l}\text { Número de empresas con } \\
\text { utilización de servicios de tra- } \\
\text { zabilidad/Número de empresas } \\
\text { totales }\end{array}$ \\
\cline { 2 - 3 } Unidad de medición & $\begin{array}{l}\text { Número de empresas con siste- } \\
\text { mas transaccionales en línea/ } \\
\text { Número de empresas totales }\end{array}$ \\
\hline Viabilidad & Porcentual \\
\hline \multirow{2}{*}{ Entidad que proporciona la información } & SI \\
\hline
\end{tabular}

Tabla 2 - Macro indicador 2 - Fuente: Propia

Entre las observaciones realizadas para la implementación de este Macro Indicador está determinar la manera como se le va a dar un valor cualitativo a los elementos que lo componen. Por lo tanto, la consolidación de este Macro Indicador, una vez obtenida la información, debe ser realizada por el Ministerio de Transporte.

\section{Vehículos con implementación de TIC para trazabili-}

dad en entregas por corredor: A continuación se presenta la información asociada a este indicador: 


\begin{tabular}{c|l} 
Forma de cálculo del Macro Indicador & Porcentual \\
\hline Unidad de medición del Macro Indicador & Porcentual \\
\hline Forma de cálculo & $\begin{array}{l}\text { Número de vehículos con tec- } \\
\text { nologías de monitoreo/Número } \\
\text { de vehículos disponibles para la } \\
\text { movilización de carga }\end{array}$ \\
\hline Unidad de medición & Porcentual \\
\hline Viabilidad & SI \\
\hline Entidad que proporciona la información & INVIAS \\
\hline Periodicidad & Anual
\end{tabular}

Tabla 3 - Macro indicador 3 - Fuente: Propia

\section{Inversión anual en TIC (sector público + privado): A}

continuación se presenta la Información asociada a este indicador:

\begin{tabular}{c|l} 
Forma de cálculo del Macro Indicador & Porcentual \\
\hline Unidad de medición del Macro Indicador & Porcentual \\
\hline Forma de cálculo & $\begin{array}{l}\text { Total de Inversión realizada al } \\
\text { Año (público+privado) en TIC/ } \\
\text { Total de inversión realizada al } \\
\text { año (público+privado) en el } \\
\text { sector logístico }\end{array}$ \\
\hline Unidad de medición & Porcentual \\
\hline Viabilidad & SI \\
\hline
\end{tabular}




\begin{tabular}{c|l}
\hline Entidad que proporciona la información & $\begin{array}{l}\text { Ministerio de Transporte, INVIAS, } \\
\text { empresas privadas, Agencia Na- } \\
\text { cional de Infraestructura }\end{array}$ \\
\hline Periodicidad & Anual
\end{tabular}

Tabla 4 - Macro indicador 4 - Fuente: Propia

\section{Porcentaje de cobertura a nivel nacional para el moni-}

toreo de mercancías: A continuación se presenta la Infor-

mación asociada a este indicador:

\begin{tabular}{c|l} 
Forma de cálculo del Macro Indicador & $\begin{array}{c}\text { Ponderación cualitativa de los } \\
\text { elementos que lo componen }\end{array}$ \\
\hline Unidad de medición del Macro Indicador & Porcentaje ponderado \\
\hline Unidad de medición & $\begin{array}{l}\text { Total de kilómetros cuadrados } \\
\text { de cobertura en el departamen- } \\
\text { to } / \text { Total de kilómetros cuadra- } \\
\text { dos del total departamental }\end{array}$ \\
\hline Viabilidad & Porcentual \\
\hline Entidad que proporciona la información & INVIAS \\
\hline Periodicidad & Anual
\end{tabular}

Tabla 5 - Macro indicador 5 - Fuente: Propia

Nuevamente, entre las observaciones realizadas para la implementación de este Macro Indicador está determinar la manera como se le va a dar un valor cualitativo a los elementos que lo componen. Por 
lo tanto, la consolidación de este Macro Indicador, una vez obtenida la información, debe ser realizada por el Ministerio de Transporte.

\section{Número de sistemas de información de servicios} logísticos en operación: A continuación se presenta la Información asociada a este indicador:

\begin{tabular}{c|l} 
Forma de cálculo del Macro Indicador & $\begin{array}{l}\text { Sumatoria de las variables aso- } \\
\text { ciadas }\end{array}$ \\
\hline Unidad de medición del Macro Indicador & Numérico \\
\hline Forma de cálculo & $\begin{array}{l}\text { Sumatoria del Número de Siste- } \\
\text { mas de Información Operativos } \\
\text { en el año }\end{array}$ \\
\hline Unidad de medición & Unidades \\
\hline Viabilidad & SI \\
\hline Entidad que proporciona la información & Ministerio de Transporte \\
\hline Periodicidad & Anual
\end{tabular}

Tabla 6 - Macro indicador 6 - Fuente: Propia

\section{Número de sistemas de información de servicios lo-} gísticos desarrollados y en fase de prueba: A continuación se presenta la Información asociada a este indicador: 


\begin{tabular}{c|l}
\hline Unidad de medición del Macro Indicador & Numérico \\
\hline Forma de cálculo & $\begin{array}{l}\text { Sumatoria del número de } \\
\text { sistemas de información en } \\
\text { desarrollo o en fases de prueba } \\
\text { en el año }\end{array}$ \\
\hline Unidad de medición & Unidades \\
\hline Viabilidad & SI \\
\hline Entidad que proporciona la información & $\begin{array}{l}\text { Ministerio de Transporte, RUNT, } \\
\text { sector privado y educativo }\end{array}$ \\
\hline Periodicidad & Anual
\end{tabular}

Tabla 7 - Macro indicador 7 - Fuente: Propia

\section{Porcentaje de transacciones de consolidación de carga} realizadas vía web: A continuación se presenta la Información asociada a este indicador:

\begin{tabular}{c|l} 
Forma de cálculo del Macro Indicador & Porcentual \\
\hline Unidad de medición del Macro Indicador & Porcentual \\
\hline Forma de cálculo & $\begin{array}{l}\text { Número de transacciones de } \\
\text { consolidación de carga vía web } \\
\text { o sistemas logísticos transaccio- } \\
\text { nales sobre el número de viajes } \\
\text { realizados en consolidación }\end{array}$ \\
\hline Unidad de medición & Porcentual \\
\hline Viabilidad & SI \\
\hline
\end{tabular}




\begin{tabular}{c|l}
\hline Entidad que proporciona la información & $\begin{array}{l}\text { Ministerio de Transporte, RNDC, } \\
\text { sector privado }\end{array}$ \\
\hline Periodicidad & Anual
\end{tabular}

Tabla 8 Macro indicador 8 - Fuente: Propia

\section{9. Índice de costos de transporte por medio de transpor-}

te: A continuación se presenta la Información asociada a este indicador:

\begin{tabular}{|c|c|}
\hline Forma de cálculo del Macro Indicador & Costo total por modo de transporte \\
\hline Unidad de medición del Macro Indicador & Numérico \\
\hline Forma de cálculo & $\begin{array}{l}\text { Costo total de los viajes realiza- } \\
\text { dos en el mes por modo de trans- } \\
\text { porte / Número de viajes realiza- } \\
\text { dos por modo de transporte }\end{array}$ \\
\hline Unidad de medición & SCOP \\
\hline Viabilidad & $\mathrm{SI}$ \\
\hline Entidad que proporciona la información & $\begin{array}{l}\text { Ministerio de Transporte, INVIAS, } \\
\text { empresas privada }\end{array}$ \\
\hline Periodicidad & Mensual \\
\hline
\end{tabular}

